\title{
Observations and Interpretation of Luminous Blue Variables
}

\author{
Henny J.G.L.M. Lamers \\ Astronomical Institute and SRON Laboratory for Space Research, \\ Princetonplein 5, NL-3584 CA Utrecht, The Netherlands, \\ hennyl@sron.ruu.nl
}

\begin{abstract}
The different types of variations of LBVs are discussed. The "typical LBV variations" have amplitudes of $\Delta V \simeq 0.5$ to 2.0 magnitudes and irregular time-scales of months to years. This is due to changes in the stellar radius and the effective temperature. Modelling of this variability for one star, $S$ Dor, shows that the radius of the star varies between 100 and $380 R_{\odot}$, the effective temperature between 20,000 and $9,000 \mathrm{~K}$, and the luminosity between $\log L_{*}=6.10$ to 5.9. The variation of the radius is not an apparent variation of the effective radius of the wind due to a variable mass loss rate (which has often been assumed) but it is a true variation of the radius of the star itself. The changes in $L_{*}$ suggest that about $10^{-3}$ to $10^{-2} M_{*}$ takes part in the expansion of the star. The irregular microvariations with amplitudes of about $\Delta V \simeq 0.2^{m}$ on timescales of weeks are probably due to non-adiabatic pulsations with modeinteraction. We argue that LBVs are close to their effective Eddington Limit and discuss a qualitative scenario to explain their location in the HR-diagram.
\end{abstract}

\section{Introduction}

Luminous Blue Variables (LBVs) form a small class of very luminous irregular photometric variables, originally called "P Cygni type stars" in our Galaxy, "S Doradus variables" in the LMC, and "Hubble Sandage variables" in other galaxies. The variability occurs on all time-scales from weeks to decades, with amplitudes of $\Delta V \simeq 0.2^{m}$ to $2^{m}$. Some LBVs have also suffered "giant eruptions" when they brightened by $3^{m}$ or more and ejected a considerable amount of mass on the order of $1 M_{\odot}$ in a few decades. The most famous examples of these are the eruptions of P Cygni around 1600 and of $\eta$ Car between 1830 and 1860. From small-number statistics together with evolutionary considerations and from the observations of ring nebulae around some LBVs it is estimated that most, if not all, LBVs suffer giant eruptions at intervals on the order of $10^{3}$ years.

Fig. 1 shows the light curve of the LBV AG Car over more than a decade. Notice the irregularity of the brightness variations without any periodicity. At best there is a "characteristic time-scale" (sometimes called "semi-period") of about 2 years and a "characteristic amplitude" of $\Delta V \simeq 1.5^{m}$ to $2.0^{m}$. These variations on a time-scale of years with amplitudes of $0.5^{m}$ to $2.0^{m}$ have been called the "typical LBV variations" or "S Dor variations" or "moderate LBV 


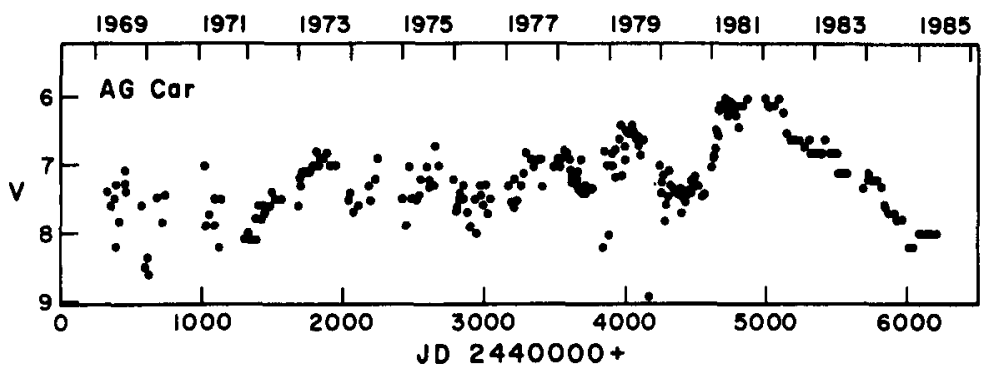

Figure 1. The visual lightcurve of the LBV AG Car between 1969 and 1985. Notice the variability on all time-scales from weeks to years. (from Lamers, 1989)

variations" (although they are not moderate at all!). I will refer to them as the typical LBV variations. Fig. 1 also shows the presence of both faster and slower variations.

LBVs represent a short post main-sequence phase of about $3.10^{4}$ years in the lives of stars with an initial mass $M \leq 50 M_{\odot}$. However, there is also a group of lower luminosity LBVs with initial masses between about 35 and 50 $M_{\odot}$. These stars might be in a post Red Supergiant phase.

In this paper I will discuss some aspects of the variability of LBVs. I will not discuss the giant eruptions although these are fascinating and crucial for the evolution of the LBVs into Wolf-Rayet stars. Instead I will concentrate on the "typical LBV variations" and on the "micro-variations" because these are possibly due to instabilities which are of relevance for stellar pulsations, the topic of this conference. I will discuss the observations and their interpretation, but I will leave the theory of the instability mechanism to be discussed by Cox et al. (these proceedings).

An excellent review about the LBVs has been published by Humphreys \& Davidson (1994). The reader is referred to that paper and to the book "Physics of Luminous Blue Variables" (Davidson et al. 1989) for topics and aspects that are not discussed here.

\section{Typical LBV variations}

\subsection{The observations}

Typical photometric LBV variations are shown in Fig. 2. The amplitude of the variations in V ranges from about $2.0^{m}$ for AG Car and R127 to $0.5^{m}$ for HD160529.

Wolf (1989) has pointed out that there is a relation between the maximum amplitude of the variations and the luminosity of the star. This relation can be used to derive extragalactic distances.

The typical LBV variations in $\mathrm{V}$ are associated with changes in the colours: the stars are bluest when they are at visual minimum and reddest at visual 

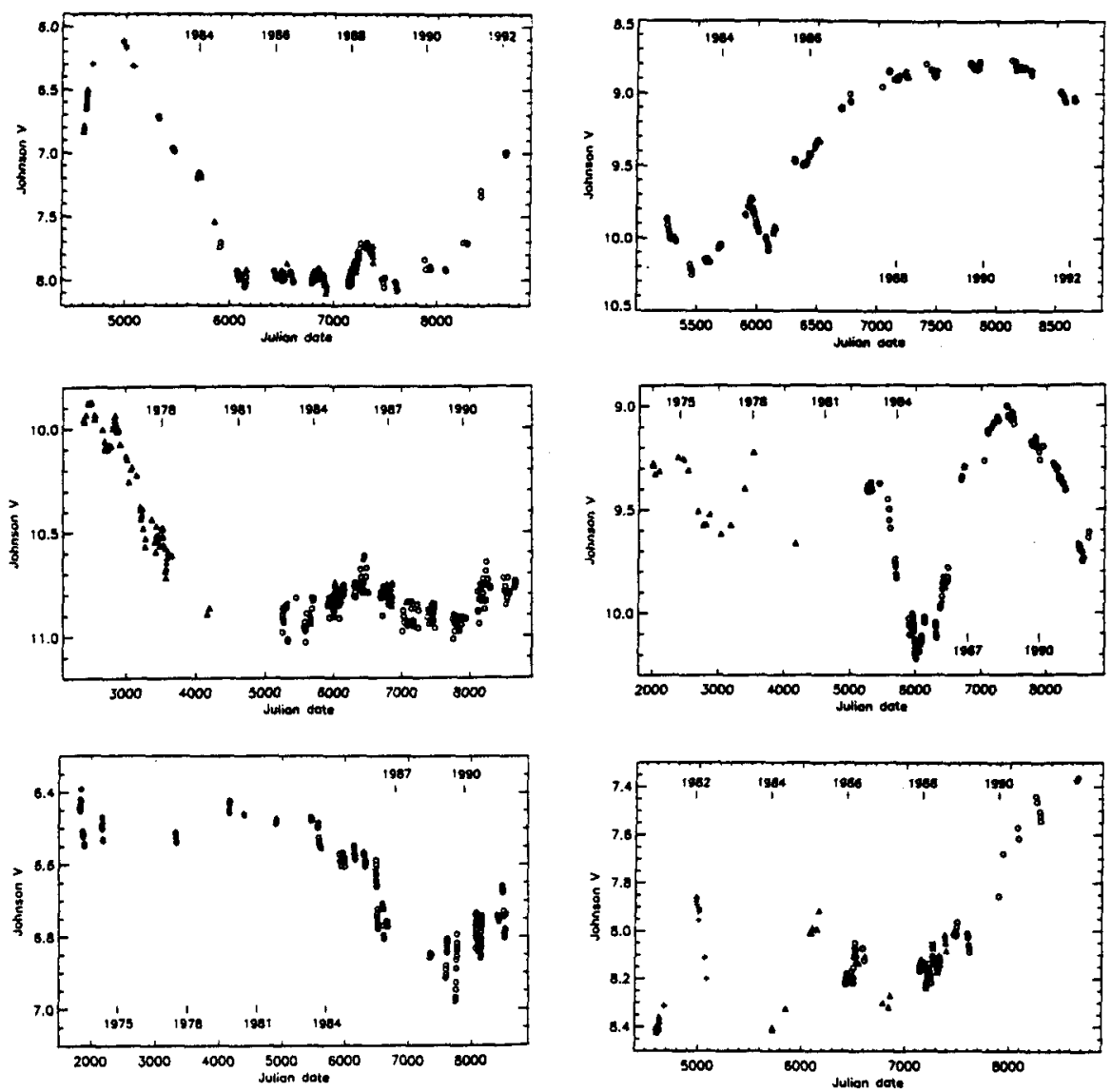

Figure 2. The visual lightcurves of six LBVs measured with the LTPV project for monitoring photometric variations of long-term variables in Strömgren-photometry. Left from top to bottom: AG Car, R71, HD160529. Right from top to bottom: R127, S Dor, HR Car. Notice the irregular nature of the "typical LBV-variations" and the fast "microvariations" during visual minimum (from Spoon et al. 1994) 
maximum. This is shown in Fig. 3 for the star R127. Between 1984 and 1988 the visual brightness increased from $10.3^{m}$ to $8.8^{m}$. The colours followed the change in $V$. These colour changes indicate that the star is hottest when it is faintest in V and coolest when it is brightest in V. This implies that the changes in $\mathrm{V}$ are largely due to changes in temperature and in bolometric correction (BC).

\subsection{Modelling the $L B V$ variations}

We have started a program in Utrecht to model the variations of LBVs in detail. The photometric variations of LBVs are modelled with the ISA wind code for modelling stars with expanding atmospheres in non-LTE (de Koter et al. 1993). This is probably the fastest non-LTE code of its kind and it can be used very efficiently to model observations of LBVs. The input of the models is: the stellar mass $M_{*}$, the radius $R_{\text {in }}$ of the star at optical depth $\tau_{\text {Ross }} \simeq 10$ to $10^{2}$, the stellar luminosity, the mass loss rate and the velocity law of the wind. In the first studies we assumed that the atmospheres consist only of $\mathrm{H}$ and He. (We are now in the process of including the metals in the models). With these input parameters we calculated the model atmospheres and the resulting energy distributions, Strömgren-colours and V-magnitude. These are compared with the observed Strömgren-photometry during the variability of LBVs.

The results for the star S Dor are shown in Fig. 4. We adopted a mass of $45 M_{\odot}$, derived from the luminosity and the models of Schaerer et al. (1993). This stellar mass is kept constant in all the models of S Dor. For mass loss rates of $\dot{M} \simeq 10^{-6}$ to $310^{-4} M_{\odot} y r^{-1}$ the energy distribution is insensitive to the mass loss rate and the velocity of the wind. This is because in this mass loss range the wind is optically thin in the visual and UV continuum (de Koter et al. 1995). Since the mass loss rates of LBVs are in the above mentioned range (e.g., Lamers, 1987) the modelling of the energy distribution and colours of LBVs is almost independent of the assumed values of $\dot{M}$ and $v_{\infty}$. The energy distribution is most sensitive to changes in the luminosity and the stellar radius, in fact to the flux $L / 4 \pi R_{\text {in }}^{2}$ and to the gravity at the inner radius of the atmosphere, i.e., to $M_{*} / R_{i n}^{2}$. A change in the gravity results in a change in the scale height of the atmosphere. A change in $L$ and $g$ results in a change in the temperature structure of the atmosphere which affects the Strömgren-colours and V directly. Therefore the Strömgren-colours and $\mathrm{V}$ are very sensitive to changes in $L$ and $R_{\text {in }}$ for a given mass.

The top part of Fig. 4 shows the variations in $\mathrm{V}$ and $u-b$. The lower panels show the derived values of $R_{\text {in }}$ and $L_{*}$ during the LBV variations and the resulting values of $T_{e f f}$ and $g_{e f f}$. The effective temperature is defined by $L=4 \pi R_{2 / 3}^{2} T_{\text {eff }}^{4}$, where $R_{2 / 3}$ is the radius at an optical depth $\tau=2 / 3$ at 5500 $\AA$. The effective gravity is $g_{\text {eff }}=G M_{*}(1-\Gamma) / R_{i n}^{2}$, where $\Gamma=g_{r a d} / g_{N}$ is the ratio between the radiation pressure force due to electron scattering and the gravity. Fig. 4 shows that the radius of the star $\mathrm{S}$ Dor changes from $100 R_{\odot}$ at visual minimum to $380 R_{\odot}$ at visual maximum. These are changes in the radius of the star itself and not only changes in the effective radius $R(\tau=2 / 3)$ of the wind. A similar change in radius was found for the LBV R71 by Leitherer et al. (1989). The effective temperature changes from $20000 \mathrm{~K}$ to $9000 \mathrm{~K}$ and the luminosity changes from $\log L=6.10$ to 5.90 between visual minimum and 

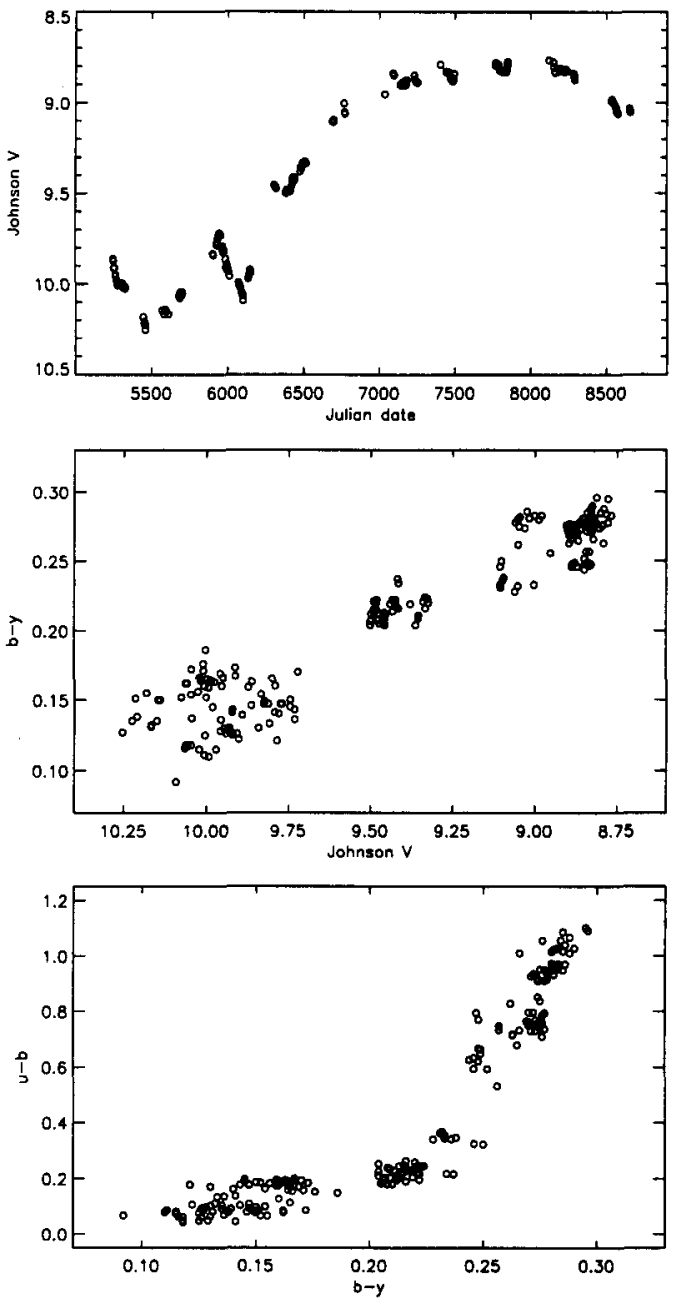

Figure 3. This figure shows the variations of the LBV R127 in the LMC in Strömgrenphotometry. Notice that there is a colour-magnitude relation and a colour-colour relation. The star is hotter when it visually faint and cooler when it is visually bright. (From de Koter \& Lamers, 1993) 

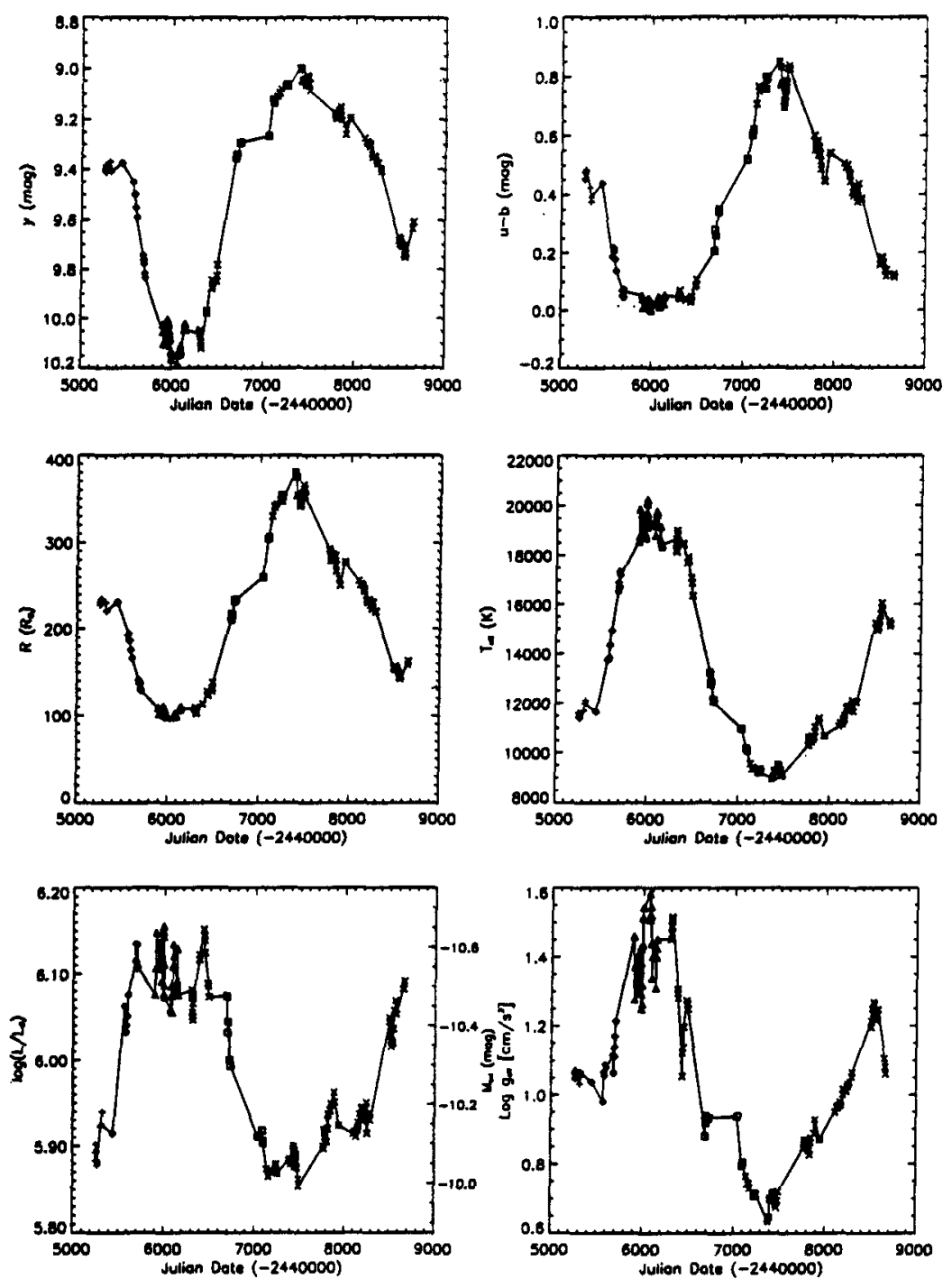

Figure 4. Results of the modelling of the photometric variations of $\mathrm{S}$ Dor. The top part shows the observed variations in magnitude and colour. The lower panels show the derived variations of the stellar parameters: $R_{*}, T_{\text {eff }}, \log \mathrm{L}$ and $\log g_{\text {eff. }}$. Notice the large variations in $R_{*}$ and $T_{\text {eff }}$ and the variation in $L_{*}$. During visual maximum the star is largest, coolest and has the lowest luminosity. (From Vennix et al. 1995) 
maximum. The bolometric magnitude decreased by $\Delta m_{\text {bol }}=0.5^{m}$ and the $\mathrm{V}$ magnitude increased by $\Delta V=1.2^{m}$. So the bolometric correction must have changed by $1.7^{m}$ between visual minimum and maximum.

The variation in $\mathrm{L}$ is particularly interesting because it is contrary to the common assumption that the luminosity of LBVs remains essentially constant during typical LBV variations. This assumption is based on observational studies of the visual and UV energy distributions of a few LBVs observed with the IUE satellite. It was found by Wolf et al. (1981) for the star R71, by Leitherer et al. (1985) for S Dor, by Stahl \& Wolf (1986) for R127, and by Lamers et al. (1989) for AG Car. However, the uncertainty of about 10 percent in the absolute calibration of IUE and the uncertainty in the extinction correction implies that the previous conclusions about the constancy of $L$ is no more accurate than about 0.2 dex at best. Our results of the detailed modelling show that for the star S Dor $M_{\text {bol }}$ does vary by about $0.5 \mathrm{mag}$. Obviously we have to check this result for more LBVs (we are presently doing this) and to improve the accuracy of the models by using fully blanketed NLTE expanding model atmospheres in the very near future (de Koter, in preparation).

If the derived variation in $\mathrm{L}$ of the LBVs turns out to be a common characteristic of LBVs it helps to understand the origin of the variations in the underlying star. This is discussed in the next section.

We conclude that the typical photometric variations of $L B V$ s are due to changes in the radius of the underlying star and that the luminosity of the $L B V$ $S$ Dor is not constant but varies by about 0.2 dex.

\subsection{The origin of the typical $\mathrm{LBV}$ variations?}

We can use the changes in the luminosity of the LBV S Dor to obtain a first order estimate of the amount of mass involved in the radial expansion of the star by a factor four. This estimate is based on simple energy considerations and is probably correct within a factor three or so.

The mean luminosity of S Dor in the period between JD +5000 to +9000 is $L \simeq 1.010^{6} L_{\odot}$ but it dropped to about $810^{5} L_{\odot}$ during the the visual maximum between JD +6800 to +8500 (see Fig. 4). This implies a decrease in luminosity by $\Delta L \simeq 210^{5} L_{\odot}$ during about 1700 days which corresponds to a missing radiative energy of $\Delta E_{\text {rad }}=\Delta L \cdot \tau \simeq 110^{47}$ erg over this time interval.

Suppose that this missing energy was used to expand the outer layers of the star from the observed radius of $100 R_{\odot}$ to $380 R_{\odot}$. Let $M_{\text {exp }}$ be the amount of mass that takes part in the expansion. Since the outer radius of the expanding layer increased its size from 100 to $380 R_{\odot}$, but the inner radius did not expand at all, we assume a mean increase in radius of the expanding layer from about 75 to $200 R_{\odot}$. If all the missing energy $\Delta E_{\text {rad }}$ was used to expand a layer of mass $M_{\text {exp }}$ from 75 to $200 R_{\odot}$ we can write

$$
\Delta E_{\text {rad }}=\frac{G M_{\text {eff }} M_{\text {exp }}}{R_{\odot}}\left\{\frac{1}{75}-\frac{1}{200}\right\}
$$

where $M_{\text {eff }}=M_{*}(1-\Gamma)$ is the effective mass of the star, i.e., corrected for the outward force due to radiation pressure, with

$$
\Gamma=g_{\text {rad }} / g_{N}=2.6610^{-5}\left(L_{*} / L_{\odot}\right) /\left(M_{*} / M_{\odot}\right)
$$


For S Dor we adopt $M_{*}=45 M_{\odot}, L_{*}=1.010^{6} L_{\odot}$ which results in $M_{\text {eff }}=$ $18 M_{\odot}$. Substitution of these values into eq. (1) gives an estimate of the amount of mass involved in the expansion

$$
M_{\text {exp }} \simeq 0.17 M_{\odot} \simeq 0.003 M_{*} .
$$

In this estimate we have neglected the thermal energy. If the star remains in thermal equilibrium, the cooling of the expanding layers also releases energy. This can increase the estimate of $M_{\text {exp }}$ by about a factor 2 .

We conclude that about 3 to $610^{-3} M_{*}$ takes part in the expansion of $\mathrm{S}$ Dor during visual maximum. This is only a small fraction of mass of the star. However, because of the very steep density profile of the early type supergiants, the expanding layer may extend to much deeper than half the stellar radius at visual minimum and deeper than $\mathbf{9 0}$ percent of the stellar radius at visual maximum.

This suggests that the typical $L B V$ variations, which are in fact due to drastic changes in the stellar radius, are due to some instability in the star which is located at about $10^{-3}$ to $10^{-2} M_{*}$ below the photosphere.

\section{The microvariations}

LBVs show microvariations of $\Delta V \simeq 0.10^{m}$ to $0.30^{m}$ on time-scales of 10 to 50 days. These variations are more pronounced at visual minimum than at visual maximum. The microvariations have been studied extensively by van Genderen (see, e.g., van Genderen 1991 and van Genderen et al. 1992). Normal supergiants in the same area of the HR diagram as the LBVs also show these types of variations but with smaller amplitudes. They are usually referred to as $\alpha$ Cygni variations. Fig. 5 shows a typical example of the microvariations of R71.

The time-scale of the microvariations is changing continuously in an irregular manner. For instance, the microvariations of R71 shown in Fig. 5 had a characteristic ("peak-to-peak") time-scale of 24 days in 1983-1985. However it changed to 14 days in 1986-1987. A recent Fourier analysis by Spoon et al. (1995) of the microvariations of LBVs has shown that the Fourier spectrum of every observing run (typically six months) is different.

Van Genderen et al. (1992) has studied the colour variations of the LBVs in the Walraven photometric system. He found that when the star is brighter in V it is bluer and hotter. This is opposite to the colour variations during the typical LBV variations! Van Genderen pointed out that temperature variations alone cannot explain both the magnitude and the colour variations and concluded that microvariations are due to changes in both temperature and radius. This suggests some kind of pulsational instability. It should be noted however that there is no phase lag between the colour variations and the brightness variations as in the Cepheids. So the variations are not simply due to irregular radial pulsations.

Gautschy (1992) has argued that the photometric variations in the supergiant $\alpha$ Cygni are due to non-adiabatic pulsations where mode-interaction is responsible for the irregular behaviour. The similarity between the photometric 


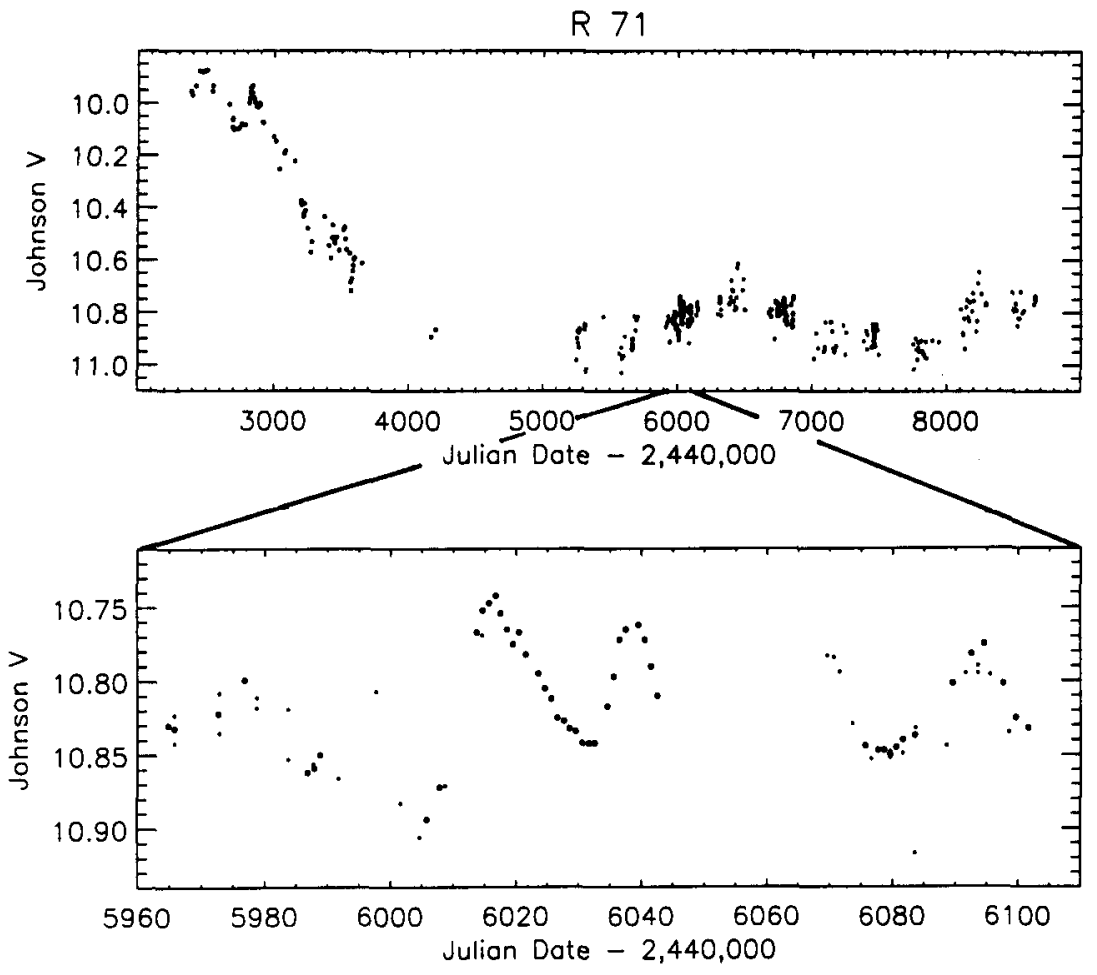

Figure 5. The lightcurve of R71 in Strömgrenphotometry measured in the LTPV-project. The upper panel shows the lightcurve over an interval of 7000 days. The error bars are on the order of 0.005 mag. The upper figure already shows the presence of short-term variability. It is shown most clearly in the expanded plot of the lower figure which covers an interval of 150 days. The "microvariations" have an amplitude of about $0.15^{m}$ and a characteristic "peak-to-peak"-time of 24 days. (From Spoon et al. 1994) 


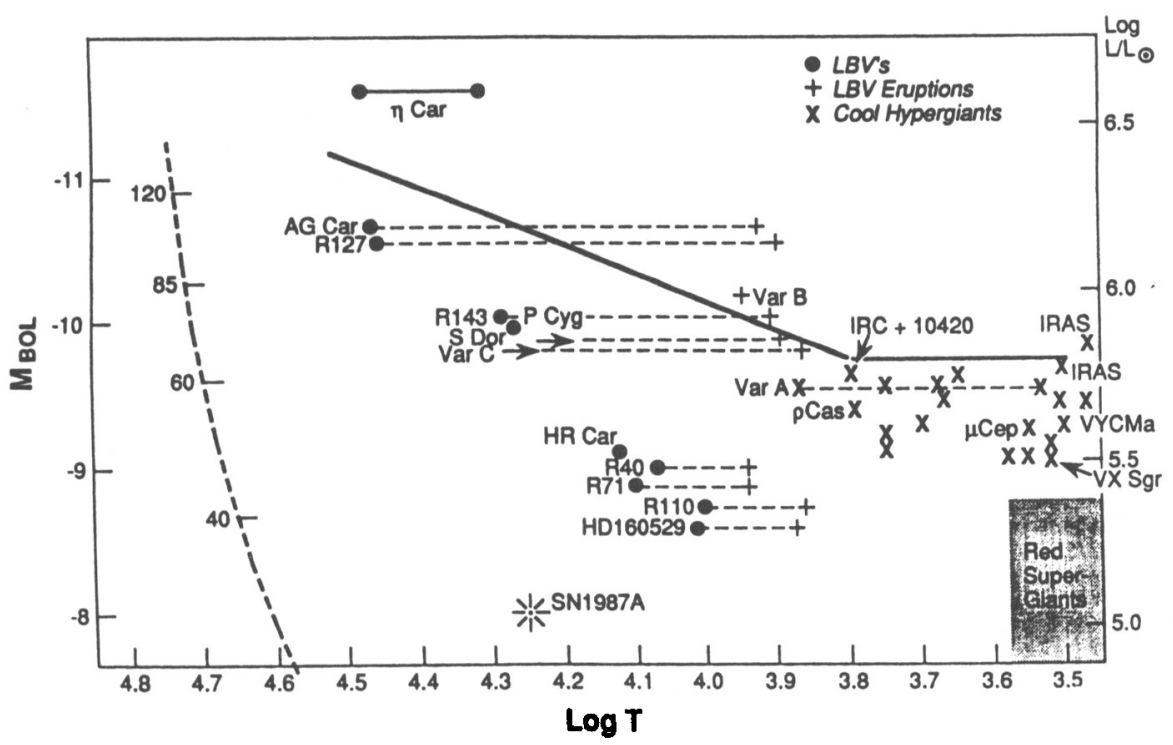

Figure 6. The location of LBVs in the HR-diagram. Each LBV is indicated by two positions, connected by horizontal dashed lines: the left side indicates the location during visual minimum, the right side during visual maximum. The location of cool hypergiants and SN 1987A is also indicated. The mainsequence with various stellar masses is shown by a thick dashed line and the Humphreys-Davidson upper limit is indicated by thick straight lines. (From Humphreys \& Davidson, 1994)

variations of $\alpha$ Cyg and the LBVs suggests that the same mechanism might also explain the microvariations of the LBVs.

\section{LBVs and the Eddington limit}

Fig. 6 shows the location of the LBVs in the HR-diagram ${ }^{1}$. The figure suggests that the luminosity is constant. However I have shown in section 2.2 that this may not be the case.

\footnotetext{
${ }^{1}$ The concept of "effective temperature" looses its meaning for stars with extended atmospheres when the radius of the photosphere, e.g., $R\left(\tau_{\lambda}=2 / 3\right)$ is a function of wavelength. In that case $T_{\text {eff }}$ is no longer defined by $T_{\text {eff }}=\left\{L / 4 \pi R_{*}^{2}\right\}^{1 / 4}$. The value of $T_{\text {eff plotted in Fig. } 6 \text { is that of }}$ a plane parallel model atmosphere that produces about the observed energy distribution
} 
At visual maximum the LBVs all reach about the same effective temperature of $T_{\text {eff }} \simeq 8000 \mathrm{~K}$. At this temperature the bolometric correction is about $0^{m}$ so $\mathrm{V}$ at visual maximum is a good indicator of $m_{b o l}$.

The LBVs at visual minimum are located in a narrow strip which runs from about $\left(\log \mathrm{L}, \log T_{\text {eff }}\right)=(6.2,4.47)$ to $(5.3,4.00)$. The process that is responsible for the LBVs must operate on stars in an evolutionary phase which corresponds to this instability strip. There are also other, i.e., more normal, supergiants in the LBV-strip. This implies that the instability of LBVs is not only related to the location in the HR-diagram but that there must be something special in the structure or the evolutionary phase that makes a star an LBV.

Humphreys \& Davidson (1984), Lamers (1986) and Appenzeller (1986) have suggested that LBVs are unstable because they are close to their effective Eddington limit. The Eddington limit is defined by $g_{r a d}=g_{N}$ where the radiation pressure force equals the force of gravity. ${ }^{2}$

This can be written in terms of an L/M ratio at the Eddington limit of

$$
\frac{L_{E}}{M_{E}}=\frac{4 \pi c G}{\kappa_{F}}
$$

The opacity in the photosphere increases with decreasing $T_{\text {eff }}$ from $40000 \mathrm{~K}$ (where most of the opacity is from electron scattering) to $10000 \mathrm{~K}$ (where the multitude of metallic lines in the Balmer continuum results in a maximum fluxmean opacity $\kappa_{F}$ ). The opacity decreases again to lower temperatures at $T_{\text {eff }} \leq$ $10000 \mathrm{~K}$.

Lamers \& Fitzpatrick (1988) have calculated the Eddington limit using the mass luminosity relation for stars at the end of the H-burning phase. Lamers \& Noordhoek (1993) have calculated the location of the Eddington limit in the HR-diagram for galaxies of different metallicities using the new opacities in the model atmospheres and in the new evolutionary tracks from Schaerer et al. (1993). They find that the Eddington limit forms a "trough" in the HR-diagram with a minimum near $10000 \mathrm{~K}$, because the opacity reaches a maximum near that temperature. The "instability strip" of the LBVs is approximately parallel to the left side of the predicted effective Eddington limit, but it does not coincide. The effective gravity of the atmospheres of LBVs is so low that they are only loosely bound to the star. It is easy to imagine that if the underlying star is unstable even a small variation may have a large effect on the loosely bound atmospheres of LBVs.

The question about the special conditions of the LBVs still has to be answered since the majority of the stars in that same region of the HR-diagram do not show the typical LBV variations. Lamers \& Fitzpatrick (1988) have argued that the LBVs have a lower mass than other stars in this region of the HR-diagram. They suggested the following scenario:

When massive stars with $M_{*} \geq 50 M_{\odot}$ evolve off the main sequence they expand and decrease in $T_{\text {eff }}$ at almost constant luminosity. They will eventually

\footnotetext{
${ }^{2}$ In the calculation of the "classical" Eddington limit only the radiation pressure by electron scattering is taken into account. In the calculation of the "effective" Eddington limit the flux mean opacity in the photosphere is taken into account. The photospheric opacity can easily be ten times larger than the electron scattering opacity
} 


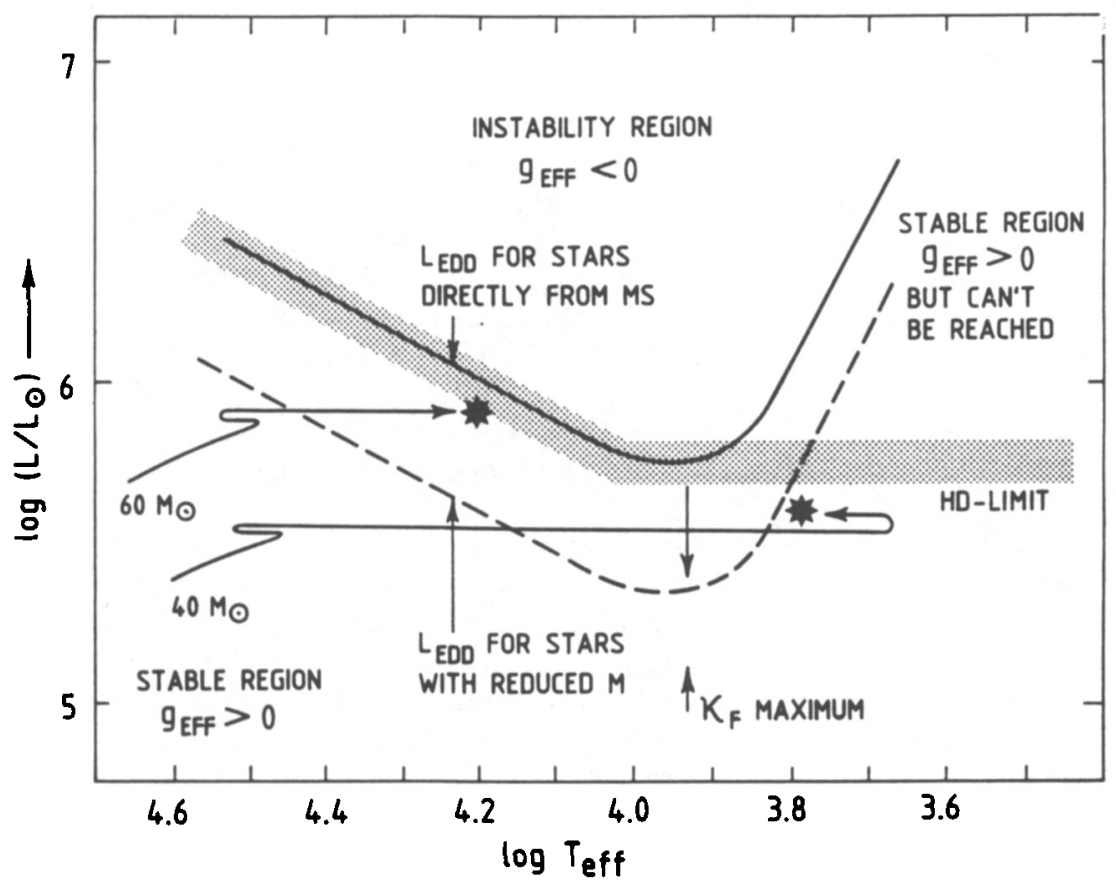

Figure 7. Schematic representation of the effect of the Eddington limit on the upper part of the HR-diagram. The Eddington limit forms a "trough" in the HR-diagram, with a minimum luminosity at $T_{\text {eff }} \simeq 8000 \mathrm{~K}$. When the star looses mass, its Eddington limit in the HR-diagram is lowered. Since massive stars evolve at almost constant luminosity the Eddington limit of a star in the post main sequence phase moves to the left in the HR-diagram when the star looses mass. However, the evolution tends to move the stars to the right. So massive stars may run frequently into "their" Eddington limit. Stars with initial mass $M_{i}<50 M_{\odot}$ will not reach their Eddington limit in the post main sequence phase but in the post red giant phase when they have lost a considerable fraction of their mass. (From de Koter, 1993) 
approach their Eddington limit. This will result in a very high mass loss rate, possibly even to large eruptions of the type of $\mathrm{P}$ Cygni or $\eta$ Car. In such eruptions as much as $1 M_{\odot}$ may be ejected. After the ejection, the star is found slightly to the left of its previous location in the HR-diagram and will evolve again to the right (Stothers \& Chin, 1979). Each phase of mass ejection will result in a decrease of the Eddington limit $L_{E}$. This is sketched in Fig. 7.

This implies that the Eddington limit for stars that evolve at constant luminosity moves to the left in the HR-diagram after each episode of high mass loss. So each star has its own Eddington limit which is determined by its present mass and luminosity. Lamers \& Fitzpatrick suggested that the LBVs are older than the other stars in the same region of the HR-diagram and that they have lost more mass. So their Eddington limit is to the left of that for normal (younger) stars. This can explain why normal stars can be found in the LBV-instability strip. This is supported by the fact that the LBVs have a higher $\mathrm{N}$-abundance in their atmosphere, which indicates that the layers which contain the products of the CNO-cycle have already reached the surface. It means that LBVs are closer to their Eddington limit than the other stars and therefore they are more unstable.

The LBVs of the lowest luminosity, with $L<10^{5.8} L_{\odot}$, are probably post red supergiant stars which are on their way to the left in the HR-diagram. When stars of $L<10^{5.8} L_{\odot}$ evolve off the main sequence they do not reach their Eddington limit because the L/M ratio of these stars is lower than for the more luminous stars. However, these stars lose a considerable fraction of their mass as red supergiants. Therefore, when they evolve back to the left in the HR-diagram their Eddington limit will be lower than in the post main sequence phase and they may still become unstable LBVs. This is supported by the calculations of the Eddington limit for post red giant stars by Lamers \& Noordhoek (1995).

Acknowledgments. I am grateful to the giraffes, zebras, rhinocerosses, impalas, baboons and louries of the Hope Game Reserve for pleasant distractions during the writing of this paper and to Frieda the ostrich for her continuous (but seldomly appreciated) interest in my work with pens and papers.

\section{References}

Appenzeller, I. 1986 in Luminous stars and Associations in Galaxies, eds. C.W.H. de Loore, A.J. Willis, P. Laskarides, Reidel, p 139

Davidson, K., Moffat, A., Lamers, H.J.G.L.M. (eds) 1989 Physics of Luminous Blue Variables, Kluwer

de Koter, A. 1993, Studies of the Variability of $L B V s$, thesis Univ. Utrecht

de Koter, A., Lamers, H.J.G.L.M. 1993 in Studies of the Variability of LBVs, thesis Univ. Utrecht, p 195

de Koter, A., Lamers, H.J.G.L.M., Schmutz, W. 1995 A\&A (in press)

de Koter, A., Schmutz, W., Lamers, H.J.G.L.M. 1993, A\&A 277, 561

Gautschy, A. 1992, MNRAS 259, 82

Humphreys, R.M. \& Davidson, K. 1994, PASP 106, 1025 
Lamers, H.J.G.L.M. 1986 in Luminous stars and Associations in Galaxies, eds. C.W.H. de Loore, A.J. Willis, P. Laskarides, Reidel, p 157

Lamers, H.J.G.L.M. 1987 in Instabilities in Early Type Stars, eds H.J.G.L.M. Lamers and C. de Loore, Reidel, p 99

Lamers, H.J.G.L.M. 1989 in Physics of Luminous Blue Variables, ed. Davidson, Moffat and Lamers, Kluwer, p135

Lamers, H.J.G.L.M., Fitzpatrick, E. 1988, ApJ 324, 279

Lamers, H.J.G.L.M., Hoekzema, N., Trams, N.R., Cassatella, A., Barylak, M. 1989 in Physics of Luminous Blue Variables, ed. Davidson, Moffat and Lamers, Kluwer, p271

Lamers, H.J.G.L.M., Noordhoek, R. 1993, in Massive Stars: their Lives in the Interstellar Medium, eds J. Cassinelli and E. Churchwell, ASP p517

Lamers, H.J.G.L.M., Noordhoek, R. 1995 (in preparation)

Leitherer, C., Appenzeller, I., Klare, G., Lamers, H.J.G.L.M., Waters, L.B.F.M., Wolf, B. 1985, A\&A 153,168

Leitherer, C., Schmutz, W., Abbott, D.C., Hamann, W.-R., Wesselowski, U. 1989, ApJ 346, 919

Maeder, A., Meynet, G. 1987, A\&A 182, 243

Schaerer, D., Charbonnel, C., Meynet, G., Maeder, A., Schaller, G. 1993, A\&AS 102,339

Spoon, H.W.W., de Koter, A., Lamers, H.J.G.L.M., Sterken, C. and Stahl, O. 1994, A\&AS 106, 141

Spoon, H.W.W., de Koter, A., Lamers, H.J.G.L.M., Sterken, C. and Stahl, O. 1995 , in preparation

Stahl, O., Wolf, B. 1986, A\&A 154, 243

Stothers, R., Chin, C.W. 1979, ApJ 233, 267

van Genderen, A.M. 1991 in Rapid Variability of OB stars, ed D. Baade, ESO Conf and Workshop Proc. Nr 36, p117

van Genderen, A.M. et al. 1992, A\&A 264, 88

Vennix, R., Lamers, H.J.G.L.M., de Koter, A. 1995 (in preparation)

Wolf, B. 1989, A\&A 217, 87

Wolf, B., Appenzeller, I., Stahl, O. 1981, A\&A 103, 94

\section{Discussion}

Pfleiderer: Expansion and contraction over about 2 A.U. within about a year should cause observable radial velocities. Do you have spectroscopic evidence not only for the expansion which might as well be stellar wind but also for the contraction phase?

Lamers: This would a very interesting independent check of the radius variations. Assuming a typical increase in the radius of about $300 R_{\odot}=210^{13} \mathrm{~cm}$ during a typical time of 2 years $=610^{7} \mathrm{~s}$, we expect photospheric expansion and contraction velocities of only about $3 \mathrm{~km} \mathrm{~s}^{-1}$. The practical problem is twofold: a) there are very few photospheric lines which are not affected by the 
stellar wind, so one has to study weak photospheric lines, b) the photospheric lines have typical widths of about $50 \mathrm{~km} \mathrm{~s}^{-1}$, so one has to look for velocity variations less than $10 \%$ of the line width for weak lines. This is presently difficult but not impossible with new high resolution, high $\mathrm{S} / \mathrm{N}$ spectra.

Habing: Your model requires rapid motion of stellar mass, travelling over large distances in short times. But could it be an apparent effect, in the sense that there is an overall increase in matter in the region between small and large radii, so that the effective stellar radius $(\tau \sim 2 / 3)$ appears to move very quickly outward ('phase velocity' against 'true velocity')?

Lamers: We have considered this possibility. An increase in the density between $100 R_{\odot}$ and $400 R_{\odot}$ can be reached in two ways: (a) either you increase the density in this range by increasing the stellar radius or (b) by increasing the density in the wind so that the layer where $\tau \sim 2 / 3$ is reached moves outward. However, this second possibility is in disagreement with the line profiles, because this requires $\dot{M}>310^{-4} M_{\odot} /$ yr whereas the emission lines and $\mathrm{P}$ Cygni profiles indicate $\dot{M} \lesssim 510^{-5} M_{\odot} /$ yr. If the star increases its radius, as in (a), by 300 $R_{\odot}$ in 2 years the velocity at the surface is not very large and only $3 \mathrm{~km} \mathrm{~s}^{-1}$. This is much smaller than the typical wind velocity of $200 \mathrm{~km} \mathrm{~s}^{-1}$.

Guzik: Could you elaborate on the assumptions that went into your energy distribution modelling with the non-LTE atmosphere code? What mass loss rate was used/implied?

Lamers: We made spherical expanding non-LTE model atmospheres of different values of $L$ and $R_{\text {in }}$ (where $R_{i n}$ is the radius of the underlying star at $\tau_{R} \sim$ 10). The mass was kept constant. The mass loss rate was also varied, but we found that this does not affect the energy distribution because the wind remains optically thin for all reasonable values of $10^{-6} \lesssim \dot{M} \lesssim 310^{-4} M_{\odot} /$ yr. So as a consequence, we could not derive the mass loss rates yet. This will come from a study of the line profiles. I guess that the mass loss rate will turn out to be several times $10^{-5} M_{\odot} / \mathrm{yr}$ at visual maximum.

Smith: Bob Wing describes pulsating stars of low gravity as "jellyfish" - you poke one somewhere and only part of the star responds. Your arguments here are predicated on spherical symmetry. For example, you infer changes in stellar radius from changes in spectral type. If, instead, a disturbance distorts a star over a confined area, the temperatures inferred from different spectral regions can be quite varied. If panchromatic observations turn out to show a variety of spectral types at one time, then it will bolster your case that the disturbance is relatively close to the star's surface because it will imply the local jellyfish response.

Lamers: You are right. We assumed spherical models so we derived the parameters of spherical LBV's. In the future we can test the spherical assumption when we include the study of the variations of the line profiles.

Percy: In the case of $\rho$ Cas, the 'micro-variations' are due to pulsation, but there are occasional large, long cycles which probably result in enhanced mass loss. Could the pulsations in LPV's also trigger 'outbursts'? 
Lamers: I doubt this. Firstly, because the typical time scale of the LPV outbursts is between 2 and 10 years, whereas the pulsations are on time scales of 10 to 40 days; secondly, the amplitudes of the pulsations are small, i.e., about 0.1 to $0.2 \mathrm{mag}$ in V; thirdly, the pulsations are probably non-radial.

Maeder: You have given a nice argument based on energy conservation to show that about 1 or $2 \%$ of the LBV mass is lifted during the outbursts. There is another line of arguments which support your result. After a major change of radius, the star re-adjusts thermally during the inter-burst phase. From the observed characteristic times, we may ask what is the thickness of a layer which has just the thermal time scale corresponding to the observed ones. Interestingly enough, this thickness also corresponds to $1-2 \%$ of the total mass. Thus, both the energy and time scale arguments are consistent.

Kovács: It is important to keep in mind that the linear adiabatic normal-mode frequencies have little connection with the observed ones for stars of extended envelopes. Non-adiabatic and nonlinear effects change the adiabatic values considerably (see Y. Tuchman's paper in these proceedings). Therefore, any conclusion about the non-radial nature of the pulsation is premature at this point (assuming that the conclusion is based solely on a period argument). 